\title{
PRINCIPAL HOMOGENEOUS SPACES AND GROUP SCHEME EXTENSIONS
}

\author{
BY \\ WILLIAM C. WATERHOUSE ${ }^{(1)}$
}

\begin{abstract}
Suppose $G$ is a finite commutative group scheme over a ring $R$. Using Hopf-algebraic techniques, S. U. Chase has shown that the group of principal homogeneous spaces for $G$ is isomorphic to $\operatorname{Ext}\left(G^{\prime}, G_{m}\right)$, where $G^{\prime}$ is the Cartier dual to $G$ and the Ext is in a specially-chosen Grothendieck topology. The present paper proves that the sheaf $\operatorname{Ext}\left(G^{\prime}, G_{m}\right)$ vanishes, and from this derives a more general form of Chase's theorem. Our Ext will be in the usual $(f p q c)$ topology, and we show why this gives the same group. We also give an explicit isomorphism and indicate how it is related to the existence of a normal basis.
\end{abstract}

0 . We begin by summarizing background results and establishing our notation; the facts here stated without proof can be found in [3], [5], [7], and [8]. Let $P$ be a prescheme, $G$ a flat commutative group scheme affine over $P$. Let $X$ be any prescheme over $P$. A principal fiber space for $G$ over $X$ is a sheaf $Y$ (for the $f p q c$ topology) with morphisms $\sigma: G \times_{P} Y \rightarrow Y$ and $p: Y \rightarrow X$ such that

(1) $G$ operates on $Y$ (via $\sigma$ ) over $X$.

(2) The map $G \times_{P} Y \rightarrow Y \times_{X} Y$ defined on $Q$-valued points by $(g, y) \mapsto(g y, y)$ is an isomorphism.

(3) The map $p$ is a sheaf epimorphism.

It is a theorem that every principal fiber space is representable; furthermore, the map $p: Y \rightarrow X$ is affine and faithfully flat. In case $X=P$ we call $Y$ a principal homogeneous space for $G$.

Suppose $P=\operatorname{Spec} R, G=\operatorname{Spec} A$, and $X=\operatorname{Spec} T$. Then $Y$ also is affine, say $Y=\operatorname{Spec} S$, and the definition is equivalent to giving maps $\sigma^{\prime}: S \rightarrow A \otimes_{R} S$ and $p^{\prime}: T \rightarrow S$ such that

(1') The map $\sigma^{\prime}$ is $T$-linear and makes $S$ an $A$-comodule.

(2') The map $\left(\sigma^{\prime}, 1 \otimes\right.$ id): $S \otimes_{T} S \rightarrow A \otimes_{R} S$ is an isomorphism.

(3') $S$ is faithfully flat over $T$.

If $T=R$ we see that these are precisely the "Galois $A$-objects" of [2].

Suppose $Y_{1}$ and $Y_{2}$ are principal fiber spaces for $G$ over $X$. Let $G$ act on $Y_{1} \times_{X} Y_{2}$ by $g\left(y_{1}, y_{2}\right)=\left(g y_{1}, g^{-1} y_{2}\right)$, and let $Y$ be the quotient sheaf. Then $Y$ is another principal fiber space, and this operation turns the set $H^{1}(X, G)$ of isomorphism classes of such spaces into an abelian group.

Received by the editors May 19, 1969 and, in revised form, February 16, 1970.

AMS 1969 subject classifications. Primary 1450, 1370; Secondary 1820.

Key words and phrases. Commutative group scheme, principal homogeneous space, line bundle, extension group, dual group scheme, Grothendieck topology, normal basis.

(1) The author was supported by an NRC-ONR research associateship.

Copyright (C) 1971, American Mathematical Society 
If a map between principal fiber spaces commutes with the $G$-actions and the projections to $X$, it is an isomorphism.

Elements of $H^{1}\left(X, G_{m}\right)$, where $G_{m}$ is the multiplicative group, are called line bundles. To study them, suppose first $X=\operatorname{Spec} T$ is affine. Then $Y=\operatorname{Spec} S$, and $\sigma^{\prime}$ is a $T$-linear map of $S$ into $S\left[u, u^{-1}\right]$. Letting $\pi_{n}(s)$ be the coefficient of $u^{n}$ in $\sigma^{\prime}(s)$, we find that the $\pi_{n}$ are a set of pairwise orthogonal projections giving a decomposition of $S$ into $T$-modules $S_{n}$; furthermore $S_{0} \simeq T$, the $T$-module $S_{1}$ is invertible, and $S_{n} \simeq S_{1}^{\otimes n}$. Every invertible $T$-module conversely gives a line bundle, and the bundle is trivial iff the module is free. Even for $X$ not affine this reasoning shows $H^{1}\left(X, G_{m}\right)=$ Pic $(X)$. Since an invertible module is locally free, we see also that every line bundle is locally trivial in the Zariski topology, i.e. there is a covering of $X$ by open affines over each of which there is a section.

Let $G$ be as above, and let

$$
0 \rightarrow G \rightarrow F \rightarrow H \rightarrow 0
$$

be a sequence of commutative group schemes over $P$. It is called exact (in fpqc) if it makes $F$ a principal fiber space for $G$ over $H$. In particular, if we begin with just a sheaf $F$ of commutative groups, we can deduce its representability. The set of isomorphism classes of such extensions is called $\operatorname{Ext}(H, G)$; by the preceding sentence this is the same as $\operatorname{Ext}^{1}(H, G)$ computed in the abelian category of $(f p q c)$ commutative group sheaves. It is thus an abelian group, and the obvious map $\operatorname{Ext}(H, G) \rightarrow H^{1}(H, G)$ is a homomorphism. The kernel of this map consists of those extensions having a scheme-theoretic section $H \rightarrow F$. These are precisely the extensions corresponding to symmetric (Hochschild) cocycles, so we have an exact sequence

$$
0 \rightarrow H_{s}^{2}(H, G) \rightarrow \operatorname{Ext}(H, G) \rightarrow H^{1}(H, G)
$$

1. We now state and prove the main theorems. If $F_{1}$ and $F_{2}$ are commutative group schemes, then for each prescheme $Q$ over $P$ we can form $\operatorname{Ext}_{Q-\mathrm{gp}}\left(F_{1 Q}, F_{2 Q}\right)$; this gives a presheaf, and we write $\operatorname{Ext}\left(F_{1}, F_{2}\right)$ for the associated sheaf. A group scheme $G$ is called finite if it is locally represented by algebras which are free modules of finite rank over the base; it is called a twisted constant group if there is an $(f p q c)$ covering in which it becomes a constant group.

THEOREM 1. Let $G$ be a commutative group scheme over $P$ which is either finite or a twisted constant group of finite type. Then $\operatorname{Ext}\left(G, G_{m}\right)=0$.

Proof. Let (*) $0 \rightarrow G_{m} \rightarrow F \rightarrow G \rightarrow 0$ be an extension over $P$; we will prove that it is trivial locally in $f p q c$. Since this will hold then for any $Q$ in place of $P$, it will follow that the whole Ext sheaf vanishes. By a first localization we may assume that $P=$ Spec $R$ is affine, and we want to find a ring $B$ faithfully flat over $R$ such that $\left(^{*}\right)$ splits over $B$. 
Suppose first that $G=\operatorname{Spec} T$ is a finite group scheme, so $T$ is a finitely generated projective $R$-module. Write $F=\operatorname{Spec} S$ as in $\S 0$. We begin by considering the sections (if any) of (*) over $R$; they correspond naturally to retractions $G_{m} \leftarrow F$, i.e. sections $R\left[u, u^{-1}\right] \rightarrow S$. Such a section is determined by giving an invertible $s \in S$ mapping onto $u$ and such that $\delta s=s \otimes s$ (where $\delta: S \rightarrow S \otimes S$ is the comultiplication corresponding to $F \times F \rightarrow F$ ). If $\varepsilon: S \rightarrow R$ is the counit, these $s$ can also be characterized as the $s \in S_{1}$ with $\delta s=s \otimes s$ and $\varepsilon(s)=1$.

Let $C=\operatorname{Hom}_{R \text {-mod }}\left(S_{1}, R\right)$. It is easy to check that $\delta$ takes $S_{1}$ to $S_{1} \otimes S_{1}$, and so $C$ has a commutative algebra structure dual to $\delta$ and $\varepsilon$. The retractions over $R$ are then precisely the maps

$$
\operatorname{Hom}_{R-\text { alg }}(C, R) \subset \operatorname{Hom}_{R-\bmod }(C, R) \simeq S_{1} .
$$

But now clearly after base extension the retractions over $B$ are

$$
\operatorname{Hom}_{B-\mathrm{alg}}\left(B \otimes_{R} C, B\right)=\operatorname{Hom}_{R \text {-alg }}(C, B) .
$$

Thus in particular there is a retraction, and hence a section over $C$; and $C$, like $S_{1}$ and $T$, is faithfully flat over $R$.

Say now $G$ is a twisted constant group; by making a faithfully flat base extension we may assume it is actually a constant group. Since we can split an extension of a direct sum if we can split each part, we may assume that $G$ is either $Z / n Z$ or $Z$. In the first case the previous argument shows that sections exist. In the second case we note that a homomorphism $\pi: F \rightarrow Z$ has a section over $B$ as soon as $1 \in Z$ (Spec $B$ ) equals $\pi(v)$ for some $v \in F(\operatorname{Spec} B)$; for there is always a unique homomorphism $Z \rightarrow F$ over $B$ taking 1 to a prescribed element in $F(\operatorname{Spec} B)$. Now since in our case $F \rightarrow Z$ is by hypothesis a sheaf epimorphism, there is a faithfully flat $B$ with $1 \in \pi F(\operatorname{Spec} B)$, and this gives us our section.

Recall that commutative group schemes $F$ and $F^{\prime}$ are called dual if there is a bilinear map $F \times F^{\prime} \rightarrow G_{m}$ inducing isomorphisms $F^{\prime} \simeq \operatorname{Hom}\left(F, G_{m}\right)$ and $F \simeq$ Hom $\left(F^{\prime}, G_{m}\right)$; here of course Hom is the sheaf assigning Hom $_{Q-\mathrm{gp}}$ to $Q$. Every finite commutative $F$ has a finite commutative dual, the Cartier dual. Twisted constant groups of finite type also have duals, called multiplicative finite type groups.

THEOREM 2. Let $G$ be a commutative group scheme over $P$ which is either finite or multiplicative finite type. Then $H^{1}(P, G) \simeq \operatorname{Ext}\left(G^{\prime}, G_{m}\right)$.

Proof. Let $\mathrm{Ext}^{n}$ be the derived functors of Hom in the category of commutative group sheaves. If we define sheaves $\mathbf{E x t}^{n}$ from them, then [10, p. V-29] the Ext ${ }^{n}$ are the derived functors of Hom. We can define $H^{n}(P, F)$ as the derived functors of $F \mapsto F(P)$, and $H^{1}(P, F)$ will be the group previously introduced. By [10, p. V-29] (cf. [6, p. 264]) we have a spectral sequence

$$
H^{p}\left(P, \mathbf{E x t}^{q}(E, F)\right) \Rightarrow \operatorname{Ext}^{p+q}(E, F)
$$


yielding in particular an exact sequence

$$
0 \rightarrow H^{1}(P, \operatorname{Hom}(E, F)) \rightarrow \operatorname{Ext}^{1}(E, F) \rightarrow H^{0}\left(P, \operatorname{Ext}^{1}(E, F)\right) .
$$

If we apply this to $G^{\prime}$ and $G_{m}$, then the map

$$
H^{1}\left(P, \operatorname{Hom}\left(G^{\prime}, G_{m}\right)\right) \rightarrow \operatorname{Ext}^{1}\left(G, G_{m}\right)
$$

is an isomorphism, since by Theorem 1 the next term in the sequence vanishes.

A similar proof of Chase's theorem has been outlined independently by $\mathrm{S}$. Shatz [9].

2. The isomorphism in Theorem 2, derived from a spectral sequence, is not particularly accessible. In this section we describe explicit maps between the two groups.

Suppose first $Y$ is a principal homogeneous space. Let $G$ act on $F_{1}=Y \times G^{\prime} \times G_{m}$ (the product over $P$, of course) by

$$
g(y, h, \alpha)=\left(g y, h,\langle g, h\rangle^{-1} \alpha\right),
$$

and let $F$ be the quotient sheaf. We map $F_{1} \times F_{1} \rightarrow F_{1}$ by

$$
(y, h, \alpha) \cdot\left(y^{\prime}, h^{\prime}, \alpha^{\prime}\right)=\left(y, h h^{\prime},\left\langle y^{-1} y^{\prime}, h^{\prime}\right\rangle \alpha \alpha^{\prime}\right) ;
$$

this is compatible with the $G$-action and so induces a map $F \times F \rightarrow F$. Thereby $F$ becomes a commutative group sheaf over $P$, the identity being induced by any $(y, 1,1)$ and the inverse of $(y, h, \alpha)$ being $\left(y, h^{-1}, \alpha^{-1}\right)$. The obvious maps $\boldsymbol{G}_{m} \rightarrow F$ and $F \rightarrow G^{\prime}$ make

$$
0 \rightarrow G_{m} \rightarrow F \rightarrow G^{\prime} \rightarrow 0
$$

exact.

Suppose now conversely that we start with $\left({ }^{*}\right)$; apply $\operatorname{Hom}\left(G^{\prime},-\right)$ to it, getting

$$
0 \rightarrow G \rightarrow \operatorname{Hom}\left(G^{\prime}, F\right) \rightarrow \operatorname{Hom}\left(G^{\prime}, G^{\prime}\right),
$$

and let $Y$ be the inverse image of id $\in \operatorname{Hom}\left(G^{\prime}, G^{\prime}\right)$. In other words, let $Y$ be the sheaf of (group) sections of $(*)$.

THEOREM 2'. These two constructions are inverse to each other, and induce isomorphisms between $H^{1}(P, G)$ and $\operatorname{Ext}\left(G^{\prime}, G_{m}\right)$.

The proof of this is mainly straightforward verification, and we will omit it. The only difficult point is showing that the sheaf of sections satisfies condition (3) for a principal fiber space, and this follows from the argument in Theorem 1.

We can give an alternate description of the first construction, one which avoids even the taking of a quotient. We take $P=\operatorname{Spec} R$, so $G=\operatorname{Spec} \mathrm{A}$ and $Y=\operatorname{Spec} S$, the action being given by a map $\sigma^{\prime}: S \rightarrow A \otimes_{R} S$. If $B$ is an $R$-algebra, we write $B^{*}$ for its group of invertible elements, and $S_{B}$ for the base extension $B \otimes_{R} S$. We recall that a sheaf is determined by its value on affine schemes; we will restrict to affine schemes and also shorten the functor notation $Y(\operatorname{Spec} B)$ to $Y(B)$. 
THEOREM 3. Define a functor by

$$
V(B)=\left\{s \in S_{B}^{*} \mid\left(\exists a \in A_{B}\right) \sigma^{\prime}(s)=a \otimes s\right\} .
$$

Map this to

$$
G^{\prime}(B)=\operatorname{Hom}\left(G, G_{m}\right)(B)=\left\{a \in A_{B}^{*} \mid \delta a=a \otimes a\right\}
$$

by sending $s$ to $a^{-1}$ if $\sigma^{\prime}(s)=a \otimes s . M a p G_{m}(B)=B^{*}$ into $V(B)$ using the natural map $B \rightarrow S_{B}$. Then

$$
0 \rightarrow G_{m} \rightarrow V \rightarrow G^{\prime} \rightarrow 0
$$

is isomorphic to the extension $\left(^{*}\right)$ associated with $Y$ in Theorem $2^{\prime}$.

Proof. Obviously $V(B \times C)=V(B) \times V(C)$. Let $B \rightarrow C$ be faithfully flat; we claim then

$$
0 \rightarrow V(B) \rightarrow V(C) \stackrel{\rightarrow}{\rightarrow} V\left(C \otimes_{B} C\right)
$$

is exact. Indeed, we know that

$$
0 \rightarrow(B \otimes S)^{*} \rightarrow(C \otimes S)^{*} \rightarrow\left(C \otimes_{B} C \otimes S\right)^{*}
$$

is exact. Suppose therefore that we have an $s \in S_{B}^{*}$ with $\sigma^{\prime}(s)=a \otimes s$ in $C \otimes A \otimes S$; we want to know that $a \in B \otimes A$. But

$$
a \otimes 1=s^{-1} \sigma^{\prime}(s) \in(C \otimes A \otimes R) \cap(B \otimes A \otimes S) ;
$$

since $S$ is faithfully flat, this intersection equals $B \otimes A \otimes R$. We have thus verified that $V$ is a sheaf.

The next step is to construct a functorial map

$$
\psi: Y(B) \times G^{\prime}(B) \times G_{m}(B) / G(B) \rightarrow V(B) ;
$$

it will then suffice to prove that when $Y(B) \neq \varnothing$ this map is a group isomorphism inducing the stated homomorphisms from $G_{m}$ and to $G^{\prime}$. To simplify notation we will take $B=R$, the general case following by base change.

We suppose then that we have an element $y \in Y(R)$, i.e., a homomorphism $y: S \rightarrow R$. Take elements $\alpha \in R^{*}=G_{m}(R)$ and $a \in G^{\prime}(R) \subset A^{*}$. Since $\left(\sigma^{\prime}, 1 \otimes \mathrm{id}\right)$ : $S \otimes S \rightarrow A \otimes S$ is an isomorphism, we can form

$$
\psi(y, a, \alpha)=(y, \mathrm{id}) \circ\left(\sigma^{\prime}, 1 \otimes \mathrm{id}\right)^{-1}(\alpha a \otimes 1) ;
$$

this is an element of $S$, invertible since $\alpha a$ is. Clearly $\psi(y, 1, \alpha)=\alpha$, so the map from $\boldsymbol{G}_{m}$ is as described.

Suppose now we take any $g: A \rightarrow R$ in $G(R)$; recall that $g y$ is the map $(g, y) \circ \sigma^{\prime}:$ $S \rightarrow R$. Consider then the commutative diagram in Figure 1. Starting with $\alpha a \otimes 1$ in $A \otimes S$ and going down first gives $\psi(g y, a, \alpha)$; going the other way gives $g(a) \psi(y, a, \alpha)=\psi(y, a, g(a) \alpha)$. Thus $\psi$ is indeed invariant under the action of $G$. 


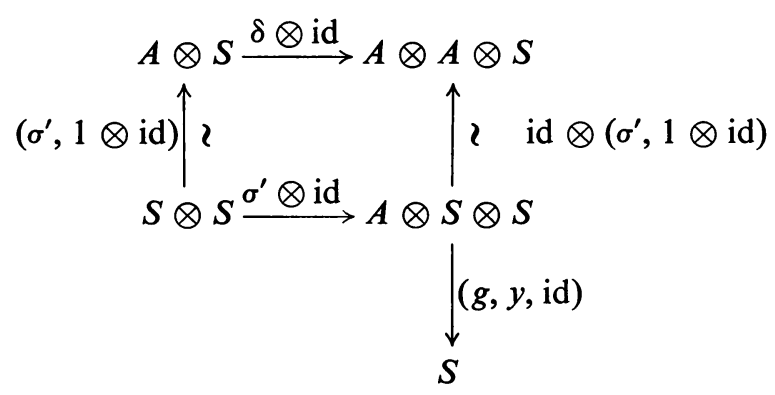

FIGURE 1

Let $s$ be any member of $V(R)$, with $\sigma^{\prime}(s)=a \otimes s$; here $a$ is invertible since $s$ is. The relation $(\delta \otimes \mathrm{id}) \sigma^{\prime}=\left(\mathrm{id} \otimes \sigma^{\prime}\right) \sigma^{\prime}$ shows that $\delta(a) \otimes s=a \otimes a \otimes s$; since $S$ is faithfully flat, $\delta(a)=a \otimes a$. Thus $a \in G^{\prime}(R)$. If we look then at the element $y(s) s^{-1} \otimes s$ in $S \otimes S$, we find that it goes to $y(s) a^{-1}$ in $A \otimes S$ and to $s$ under $\left(y\right.$, id). Thus $s=\psi\left(y, a^{-1}, y(s)\right)$, and all of $V(R)$ is in the image of $\psi$.

Next we observe that the map $G \times Y \rightarrow G \times Y$ given by

$$
(g, z) \mapsto g z \mapsto(y, g z) \mapsto\left(y(g z)^{-1}, g z\right)
$$

is also given by

$$
(g, z) \mapsto(g, y, z) \mapsto\left(g, y z^{-1}, z\right) \mapsto\left(g, g^{-1}, y z^{-1}, z\right) \mapsto\left(g^{-1}\left(y z^{-1}\right), g z\right) .
$$

Hence the corresponding composite maps $A \otimes S \rightarrow A \otimes S$ are equal. Going the first way from $\alpha a \otimes 1$ yields $\sigma^{\prime} \psi(y, a, \alpha)$; going the other way yields $a^{-1} \otimes \psi(y, a, \alpha)$. Thus $\psi$ does map into $V(R)$, and the map to $G^{\prime}$ is as described.

It is easy now to show that $\psi$ is injective. For suppose $\psi(y, a, \alpha)=\psi\left(y^{\prime}, a^{\prime}, \alpha^{\prime}\right)$. Then $a=a^{\prime}$, since we can recover $a$ from $\sigma^{\prime} \psi(y, a, \alpha)$. Using the action of $G(R)$ we may assume $y=y^{\prime}$. But $\psi(y, a, \alpha)=\alpha \psi(y, a, 1)$, and these are distinct for distinct $\alpha$.

Finally we verify that $\psi$ is a group homomorphism. Take two elements $(y, a, \alpha)$ and $\left(y^{\prime}, a^{\prime}, \alpha^{\prime}\right)$; using the $G$-action we may assume $y=y^{\prime}$. Their product then is $\left(y, a a^{\prime}, \alpha \alpha^{\prime}\right)$. But since the maps used in defining $\psi$ are algebra morphisms, we indeed have $\psi\left(y, a a^{\prime}, \alpha \alpha^{\prime}\right)=\psi(y, a, \alpha) \psi\left(y, a^{\prime}, \alpha^{\prime}\right)$.

REMARK. After eliminating some dualizations in [2], we find that $V$ is precisely the functor constructed there. Working directly with the algebras, however, Chase naturally maps $s$ to $a$ rather than to $a^{-1}$. Hence the homomorphism he constructs is the negative of ours.

3. Our next goal is to show that the Ext in Theorem 2 can be computed in a coarse Grothendieck topology. For convenience we will continue to regard our sheaves as functors on affine schemes. The basic tool is the following general result:

Proposition 1. Let $\mathscr{S}$ and $\mathscr{T}$ be two Grothendieck topologies. Suppose that

$$
0 \rightarrow A \rightarrow B \rightarrow C \rightarrow 0
$$


is a sequence of commutative group functors which are sheaves in $\mathscr{S}$, and that the sequence is exact in $\mathscr{S}$. Then if $A$ and $C$ are sheaves for $\mathscr{T}$, so is $B$.

Proof. Let $\left\{U_{i} \rightarrow W\right\}$ be a covering in $\mathscr{T}$. We first show that

$$
B(W) \rightarrow \prod B\left(U_{i}\right)
$$

is injective. Indeed, suppose an element $b$ goes to zero. Then its image in $C(W)$ goes to zero in $\Pi C\left(U_{i}\right)$ and hence equals zero. By exactness then $b$ comes from an $a \in A(W)$. But all the maps $A\left(U_{i}\right) \rightarrow B\left(U_{i}\right)$ are injective, so $a$ goes to zero in $\prod A\left(U_{i}\right)$ and hence equals zero; thus $b=0$.

Suppose now we have elements $b_{i} \in B\left(U_{i}\right)$ such that $b_{i}$ and $b_{j}$ have the same image $b_{i j}$ in $B\left(U_{i} \times U_{j}\right)$-here and throughout the proof, $\times$ stands for the product over $W$. We must produce an element $b \in B(W)$ yielding all the $b_{i}$. This is a diagramchasing argument, and the reader is encouraged to write out any diagram he feels the urge to chase.

Let $c_{i} \in C\left(U_{i}\right)$ be the image of $b_{i}$. Then $c_{i}$ and $c_{j}$ have the same image in $C\left(U_{i} \times U_{j}\right)$, so there is an element $c \in C(W)$ giving every $c_{i}$. By $\mathscr{S}$-exactness we can find a covering $\left\{V_{\lambda} \rightarrow W\right\}$ in $\mathscr{S}$ such that $c$ is in the image of $B$ there; that is, for each $\lambda$, the image of $c$ in $C\left(V_{\lambda}\right)$ comes from some $b_{\lambda} \in B\left(V_{\lambda}\right)$.

Fix $\lambda$, and consider the images of $b_{\lambda}$ and $b_{i}$ in $B\left(V_{\lambda} \times U_{i}\right)$. They become the same in $C\left(V_{\lambda} \times U_{i}\right)$, so their difference comes from an $a_{\lambda i} \in A\left(V_{\lambda} \times U_{i}\right)$. Now $b_{i}$ and $b_{j}$ become the same in $B\left(U_{i} \times U_{j}\right)$; therefore $a_{\lambda i}$ and $a_{\lambda j}$ have the same image in $B\left(V_{\lambda} \times U_{i} \times U_{j}\right)$, and hence also in $A\left(V_{\lambda} \times U_{i} \times U_{j}\right)$. Since $\left\{V_{\lambda} \times U_{i} \rightarrow V_{\lambda}\right\}$ is a covering in $\mathscr{T}$, and $A$ is a $\mathscr{T}$-sheaf, there is an $a_{\lambda} \in A\left(V_{\lambda}\right)$ giving rise to the $a_{\lambda i}$. Replacing $b_{\lambda}$ by $b_{\lambda}+a_{\lambda}$, we may assume that $b_{\lambda}$ and $b_{i}$ have the same image in $B\left(V_{\lambda} \times U_{i}\right)$.

We observe now that $b_{\lambda}$ and $b_{\mu}$ have the same image (= that of $b_{i}$ ) in $B\left(V_{\lambda} \times V_{\mu} \times U_{i}\right)$. Since we already know that

$$
B\left(V_{\lambda} \times V_{\mu}\right) \rightarrow \prod_{i} B\left(V \times V_{\mu} \times U_{i}\right)
$$

is injective, we see that $b_{\lambda}$ and $b_{\mu}$ have the same image in $B\left(V_{\lambda} \times V_{\mu}\right)$. Hence there is an element $b \in B(W)$ giving every $b_{\lambda}$. Since $b$ and $b_{i}$ have the same image (= that of $\left.b_{\lambda}\right)$ in all $B\left(V_{\lambda} \times U_{i}\right)$, the $b_{i}$ must come from $b$.

We now take $\mathscr{S}$ to be the following Grothendieck topology, used in [2]: Let Spec $C \rightarrow$ Spec $B$ be a covering if $C=\left(\prod R_{x_{i}}\right) \otimes B$, where $\sum x_{i}=1$ and the $x_{i}$ are not in the Jacobson radical of $R$. Clearly this is much coarser than the (fpqc) topology. It is fine enough for our purposes, however, because it trivializes enough line bundles.

Proposition 2. Let $Y$ be a line bundle over Spec $D$, where $D$ as an $R$-module is projective of finite type. Then there is an $\mathscr{S}$-covering in which $Y$ becomes trivial. 
Proof. In view of the remarks in $\S 0$, the proposition is equivalent to the following statement: If $M$ is any invertible $D$-module, there are $x_{1}, \ldots, x_{n}$ in $R \mid \operatorname{Rad}(R)$ with $\sum x_{i}=1$ and each $M_{x_{i}}$ free over $D_{x_{i}}$. This is straightforward commutative algebra, mostly available in $[1$, p. 65], and we just sketch the proof.

For each maximal ideal $m$ of $R$, the ring $D_{m}$ is semilocal, and hence $M_{m}$ is free. Then there is an $f \in R \mid m$ with $M_{f}$ free over $D_{f}$. The collection of all such $f$ generates an ideal lying in no maximal ideal, so there is a relation $1=\sum r_{j} f_{j}$. Dropping the terms which lie in $\operatorname{Rad}(R)$ we get a sum $g=\sum r_{i} f_{i}$ with $g$ lying in $1-\operatorname{Rad}(R)$ and hence invertible. Set $x_{i}=g^{-1} r_{i} f_{i}$.

Let $H$ now be a finite commutative group scheme over $R$. If

$$
0 \rightarrow G_{m} \rightarrow F \rightarrow H \rightarrow 0
$$

is exact, i.e. exact in $(f p q c)$, then it is exact in $\mathscr{S}$; for Proposition 2 shows that $F \rightarrow H$ is an $\mathscr{S}$-epimorphism. Conversely, if it is exact in $\mathscr{S}$, Proposition 1 shows that $F$ is a sheaf in $(f p q c)$; and of course the sequence is still exact there. Thus we have

THEOREM 4. If $H$ is a finite commutative group scheme over $R$, then $\operatorname{Ext}\left(H, G_{m}\right)$ is canonically isomorphic to the group $\operatorname{Ext}\left(H, G_{m}\right)$ computed in $\mathscr{S}$.

It is perhaps worth mentioning that one cannot here replace $\boldsymbol{G}_{m}$ by an arbitrary group. For example, let $R$ be a field of characteristic $p>0$, and consider

$$
0 \rightarrow \alpha_{p} \rightarrow \alpha_{p^{2}} \rightarrow \alpha_{p} \rightarrow 0
$$

which is exact. Since $\mathscr{S}$ has no coverings, exactness in $\mathscr{S}$ would mean exactness as presheaves, and the final map is not surjective when evaluated on $\operatorname{Spec}\left(R[u] / u^{p}\right)$.

4. Take $G$ again to be a finite commutative group scheme, and assume for simplicity that $P=\operatorname{Spec} R$. Combining Theorem 2 with a remark in $\S 0$, we find that there is an exact sequence

$$
0 \rightarrow H_{s}^{2}\left(G^{\prime}, G_{m}\right) \rightarrow H^{1}(P, G) \rightarrow \operatorname{Pic}\left(G^{\prime}\right) .
$$

In this section we will give a more explicit description of the map to Pic $\left(G^{\prime}\right)$. Let $G=\operatorname{Spec} A$, so $G^{\prime}=\operatorname{Spec} A^{\prime}$ where $A^{\prime}=\operatorname{Hom}_{R \text {-mod }}(A, R)$.

Set $T=A^{\prime}$ in the proof of Theorem 1 ; by Theorem $2^{\prime}$ we have $Y=\operatorname{Spec} \mathrm{C}$ there. Look first at the $G$-action induced on the sheaf of retractions. It is given simply by letting elements $a^{\prime} \in G(B) \subset B \otimes A^{\prime}$ act on $s \in Y(B) \subset B \otimes S_{1}$ by multiplication. This means that the action $C \rightarrow A \otimes C$ yields an $A^{\prime}$-module structure agreeing with the one naturally induced on

$$
C=\operatorname{Hom}_{R-\bmod }\left(S_{1}, R\right) .
$$

Then as an $A^{\prime}$-module,

$$
\begin{aligned}
S_{1}=\operatorname{Hom}_{R-\bmod }(C, R) & =\operatorname{Hom}_{A^{\prime}-\bmod }(C, A) \\
& =A \otimes_{A^{\prime}} C^{\vee},
\end{aligned}
$$

where $C^{\vee}$ is the inverse of $C$ in $\operatorname{Pic}\left(A^{\prime}\right)$. 
So far, however, we have been looking at the sheaf of retractions. Our actual map takes the sheaf of sections, giving the inverse principal homogeneous space (same $C$, but different action). Thus going back from $C$ we get the inverse of the above class, and we have

Theorem 5. The map $H^{1}(P, G) \rightarrow$ Pic $\left(G^{\prime}\right)$ sends $\operatorname{Spec} C$ to the class of $C \otimes_{A^{\prime}} A^{\vee}$, where $A^{\vee}$ is the inverse of $A$ as an $A^{\prime}$-module. In particular, the kernel of the map consists of those spaces for which $C$ is isomorphic to $A$ as an $A^{\prime}$-module.

Remarks. 1. If we replace $C, A$, and $A^{\prime}$ by the corresponding locally free sheaves, we can extend the theorem to nonaffine base preschemes.

2. If Pic $R=0$, or if $G$ comes by base extension from such a ring, then $A$ is a free $A^{\prime}$-module [2, p. 68]; hence in those cases the kernel is those $C$ which are free over $A^{\prime}$. This holds in particular if $G$ is a finite constant group. In the case $G=Z / n Z$, Theorem 2 and this fact were proved by H. Epp [4].

3. When $G$ is a constant group, $A^{\prime}$ is the group algebra, and to say $C$ is free is to say that it has a normal basis. At first glance one would be inclined to use this definition in general. But Theorem 5 shows that it may be better to say $C$ has a normal basis if $C$ is isomorphic to $A$ as an $A^{\prime}$-module. With this convention we can then conclude that the spaces with a normal basis form a subgroup canonically isomorphic to $H_{s}^{2}\left(G^{\prime}, G_{m}\right)$.

\section{REFERENCES}

1. S. U. Chase and A. Rosenberg, Amitsur cohomology and the Brauer group, Mem. Amer. Math. Soc. No. 52 (1965), 34-79. MR 33 \#4119.

2. S. U. Chase and M. E. Sweedler, Hopf algebras and Galois theory, Lecture Notes in Math., no. 97, Springer-Verlag, Berlin, 1969.

3. M. Demazure, A. Grothendieck et al., Schémas en groupes. Fasc. 1, Exposés 1 à 4, Séminaire de Géometrie Algébrique, 1963, Inst. Hautes Études Sci., Paris, 1963/64. MR 34 \#7517.

4. H. Epp, Commutative group schemes, Harrison's theorem, and Galois extensions, Thesis, Northwestern University, 1966, Dissertation Abstracts 27 B (1967). Abstract \#3595.

5. P. Gabriel et al., Groupes algébriques, Séminaire Heidelberg-Strasbourg, 1965/66.

6. R. Godement, Topologie algébrique et théorie des faisceaux, Actualités Sci. Indust., no. 1252, Hermann, Paris, 1958. MR 21 \#1583.

7. A. Grothendieck, Revêtements étales et groupe fondamental. Fasc. 2, Exposé 8, Séminaire de Géometrie Algébrique, 1960/61, Inst. Hautes Études Sci., Paris, 1963. MR 36 \#179b.

8. F. Oort, Commutative group schemes, Lecture Notes in Math., no. 15, Springer-Verlag, Berlin, 1966. MR 35 \#4229.

9. S. Shatz, Principal homogeneous spaces for finite group schemes, Proc. Amer. Math. Soc. 22 (1969), 678-680.

10. J. Verdier, Cohomologie étale des schémas, Séminaire de Géométrie Algébrique, 1963, Inst. Hautes Études Sci., Paris, 1963/64.

CORNELl UNIVERSITY, ITHACA, NEW YORK 14850 